\title{
Liver Transplantation after Successful Downstaging with Hepatic Arterial Infusion Chemotherapy in a Patient with Hepatocellular Carcinoma with Portal Vein Tumor Thrombus
}

\author{
Hee Chul Nam ${ }^{1,2}$, Pil Soo Sung ${ }^{1,2}$, Ho Jong Chun ${ }^{3}$, Dong Goo Kim4, Jeong Won Jang ${ }^{1,2}$, Jong Young Choi' ${ }^{1,2}$, \\ Seung Kew Yoon ${ }^{1,2}$ \\ 'Department of Internal Medicine, ${ }^{2}$ The Catholic Liver Research Center, Departments of ${ }^{3}$ Radiology and ${ }^{4}$ Surgery, College of Medicine, \\ The Catholic University of Korea, Seoul, Korea
}

Received Jan. 31, 2019

Revised Feb. 11, 2019

Accepted Feb. 11, 2019
Hepatocellular carcinoma (HCC) is one of the most common cancers worldwide. The majority of patients with $\mathrm{HCC}$ are diagnosed at advanced disease stages with vascular invasion, where curative approaches are often not feasible. Currently, sorafenib is the only available standard therapy for HCC with portal vein tumor thrombosis (PVTT). However, in many cases, sorafenib therapy fails to achieve satisfactory results in clinical practice. We present a case of advanced HCC with PVTT that was treated with hepatic arterial infusion chemotherapy (HAIC) followed by liver transplantation. Three cycles of HAIC treatment resulted in necrotic changes in most of the tumors, and PVTT was reduced to an extent at which liver transplantation was possible. Further studies are required to determine the treatment strategies for advanced HCC with PVTT that can improve prognosis. (J Liver Cancer 2019;19:64-68)

Keywords: Hepatocellular carcinoma; Venous thrombosis

\section{INTRODUCTION}

Hepatocellular carcinoma (HCC) is the fifth most common cancer and the second most frequent cause of cancerrelated death worldwide. ${ }^{1}$ Unlike other malignancies, the incidence and mortality rates of HCC will continue to increase over the next 20 years in some countries. ${ }^{2}$

Portal vein tumor thrombosis (PVTT) is commonly accomaccompanied by advanced HCC. A substantial proportion of patients with HCC present with PVTT either at the

\footnotetext{
Corresponding author : Seung Kew Yoon

Department of Internal Medicine, College of Medicine, The Catholic University of Korea, 222 Banpo-daero, Seocho-gu, Seoul 06591, Korea Tel. +82-2-2258-2073, Fax. +82-2-3481-4025

E-mail; yoonsk@catholic.ac.kr

https://orcid.org/0000-0002-4476-4868
}

onset of disease or as a result of HCC recurrence or progression, and their prognosis tends to be poor. Sorafenib has been proven to be the standard therapy for HCC with PVTT, according to most guidelines. ${ }^{1,3-5}$ However, the survival benefits of sorafenib administration are limited for patients from the Asia Pacific region. ${ }^{6,7}$

Some previous studies have reported favorable results associated with the application of hepatic arterial infusion chemotherapy (HAIC) in advanced HCC. ${ }^{8-11}$ Therefore, we report a case of liver transplantation after successful downstaging with HAIC in a patient with HCC and PVTT.

\section{CASE REPORT}

A 54-year-old man visited Seoul St. Mary's Hospital in September 2013. He complained of dull and persistent ab- 
dominal discomfort in the right upper quadrant area for several months; however, the discomfort was tolerable and unrelated to postural changes. His blood pressure was 120/85 $\mathrm{mmHg}$, heart rate was 75 beats per minute, and body temperature was $36.5^{\circ} \mathrm{C}$ at the time of admission. He was not taking any medications. He was a carrier of hepatitis B virus (HBV) but had not been undergoing regular surveillance. Further, he had been drinking approximately $108 \mathrm{~g}$ of alcohol ( 2 bottles of Soju) per day for the last 25 years.

The results of the laboratory tests were as follows: white blood cell count, 6,320 cells/ $\mu \mathrm{L}$; serum hemoglobin concentration, $13.9 \mathrm{~g} / \mathrm{dL}$; serum platelet count, $28.9 \times 10^{4}$ platelets $/ \mu \mathrm{L}$; serum aspartate aminotransferase, $70 \mathrm{IU} / \mathrm{L}$; serum alanine aminotransferase, $64 \mathrm{IU} / \mathrm{L}$; serum alkaline phosphatase, 129 IU/L; serum gamma glutamic transpeptidase, 213 IU/L; total serum bilirubin, $1.0 \mathrm{mg} / \mathrm{dL}$; serum albumin, $4.0 \mathrm{~g} / \mathrm{dL}$; and prothrombin time, $95 \%$. The patient showed positive results for hepatitis B surface antigen and hepatitis B e antibody with low levels of HBV DNA (210 copies/mL), and negative results for hepatitis B e antigen and hepatitis C antibody. The levels of serum alpha-fetoprotein (AFP) and proteins induced by vitamin $\mathrm{K}$ absence or antagonist-II (PIVKA-II) were markedly elevated at $36,070 \mathrm{ng} / \mathrm{mL}$ and $285 \mathrm{mAU} / \mathrm{mL}$, respectively.

A liver dynamic computed tomography (CT) scan demonstrated a large infiltrative-type HCC in the right posterior hepatic section. A liver magnetic resonance imaging (MRI) was performed for a more precise evaluation of the tumor char- acteristics. The mass was heterogeneously enhanced in the arterial phase, became washed out during the portal venous phase, and was identified as a defect in the hepatobiliary phase. The results of liver dynamic CT and MRI were consistent with a definitive diagnosis of HCC, according to the diagnostic criteria. ${ }^{12}$ The largest tumor size of the HCC was $11 \times 10 \mathrm{~cm}$, and numerous daughter nodules were identified around the main tumor. The tumor was accompanied by portal vein thrombus, and the thrombus extended up to the right main portal vein (Fig. 1). The thrombus was identified as the tumor thrombus, which induced expansion of the involved vessel and showed enhancement during the arterial phase. Chest CT and positron emission tomography/CT scans did not reveal any visible distant metastasis.

The patient was diagnosed with advanced HCC with vascular invasion, with a liver function of Child-Pugh class A and Eastern Cooperative Oncology Group performance status of 0 , as well as a Barcelona Clinic Liver Cancer (BCLC) tumor stage of C. According to the BCLC, the standard treatment for the patient was sorafenib; however, we decided to treat him using HAIC, based on the consensus reached in multidisciplinary meetings.

Prior to HAIC, a catheter was implanted into the tumorfeeding artery identified through angiography. A chemotherapeutic agent was administered through this catheter. The chemotherapeutic regimen used in Seoul St, Mary's hospital consists of $500 \mathrm{mg} / \mathrm{m}^{2}$ of 5 -Fluorouracil (5-FU) on days 1-3, with $60 \mathrm{mg} / \mathrm{m}^{2}$ of cisplatin on day 2. This patient did not
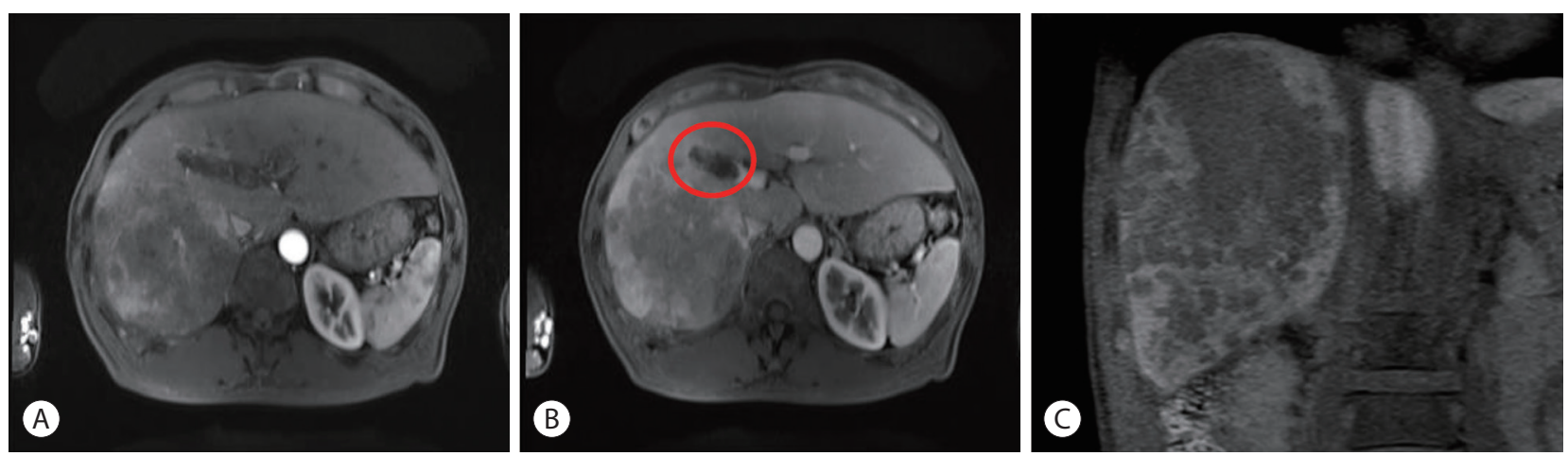

Figure 1. Liver magnetic resonance imaging (MRI) findings. (A) Arterial phase, (B) portal venous phase, (C) hepatobiliary phase. MRI revealed a large infiltrative-type hepatocellular carcinoma in the right posterior hepatic section. The tumor showed heterogeneous enhancement pattern in the arterial phase (A). Portal venous tumor thrombus was observed in the portal venous phase (circle), extending up to the right main portal vein (B). Numerous daughter nodules were found as defects around the main tumor (C). 
show any complications or adverse events after the first cycle of HAIC treatment, and the therapy was well-tolerated.

Serum tumor markers decreased drastically at 4 weeks after the first treatment. The levels of serum AFP and PIVKAII were markedly decreased to $578 \mathrm{ng} / \mathrm{mL}$ and $17 \mathrm{mAU} / \mathrm{mL}$, respectively. HAIC treatment was considered effective in this patient, and two more cycles of treatment were performed with an interval of 4 weeks.

After three cycles of HAIC treatment, the serum tumor markers of the patient decreased to a normal range. Serum AFP and PIVKA-II levels improved to $4.7 \mathrm{ng} / \mathrm{mL}$ and $15 \mathrm{mAU} / \mathrm{mL}$, respectively. A follow-up liver MRI demonstrated that the largest diameter of the mass was significantly reduced to 4 $\mathrm{cm}$; arterial enhancement was observed only in the periphery of the tumor and internal necrosis was noted (Fig. 2). The degree of the PVTT, initially extending to the right main portal vein, was also reduced to within the right posterior section of the portal vein. It was difficult to distinguish whether the thrombosis inside the portal vein was due to a viable tumor or bland thrombus.

Marginal viable portions remaining around the necrotic tumor and the significant decrease in serum tumor markers indicated successful downstaging. Liver transplantation (LT) was decided as a curative treatment following discussions at the multidisciplinary meetings. Living-donor LT was performed on February 18, 2014. The histologic findings of the resected liver were as follows. The size of the entire tumor was $4.0 \times 3.5 \mathrm{~cm}$, but the viable portion was $0.04 \times 0.04 \mathrm{~cm}$. Most of the tumors showed necrotic changes. Tumor cell differentiation was identified as Edmondson grade II, and no tumor cells were found in the portal vein. In the non-tumor tissue, septal fibrosis was observed with moderate lobular activity by HBV. There were no complications or HCC recurrence after LT during the follow-up period of 63 months.

\section{DISCUSSION}

Here, we report the successful outcome of HAIC treatments for HCC with PVTT. Although the patient had an initial infiltrative tumor that was larger than $11 \mathrm{~cm}$ with Vp3 PVTT, he could undergo LT after downstaging by three repeated cycles of HAIC. According to the meta-analysis by Parikh et al. ${ }^{13}$, the success rate of downstaging is below $50 \%$ for patients with HCC beyond the Milan criteria. In cases of HCC with vascular invasion, the success rate of downstaging is further reduced. Therapies such as conventional transarterial chemoembolization, transarterial radioembolization, sorafenib, and HAIC with or without external radiation ther-
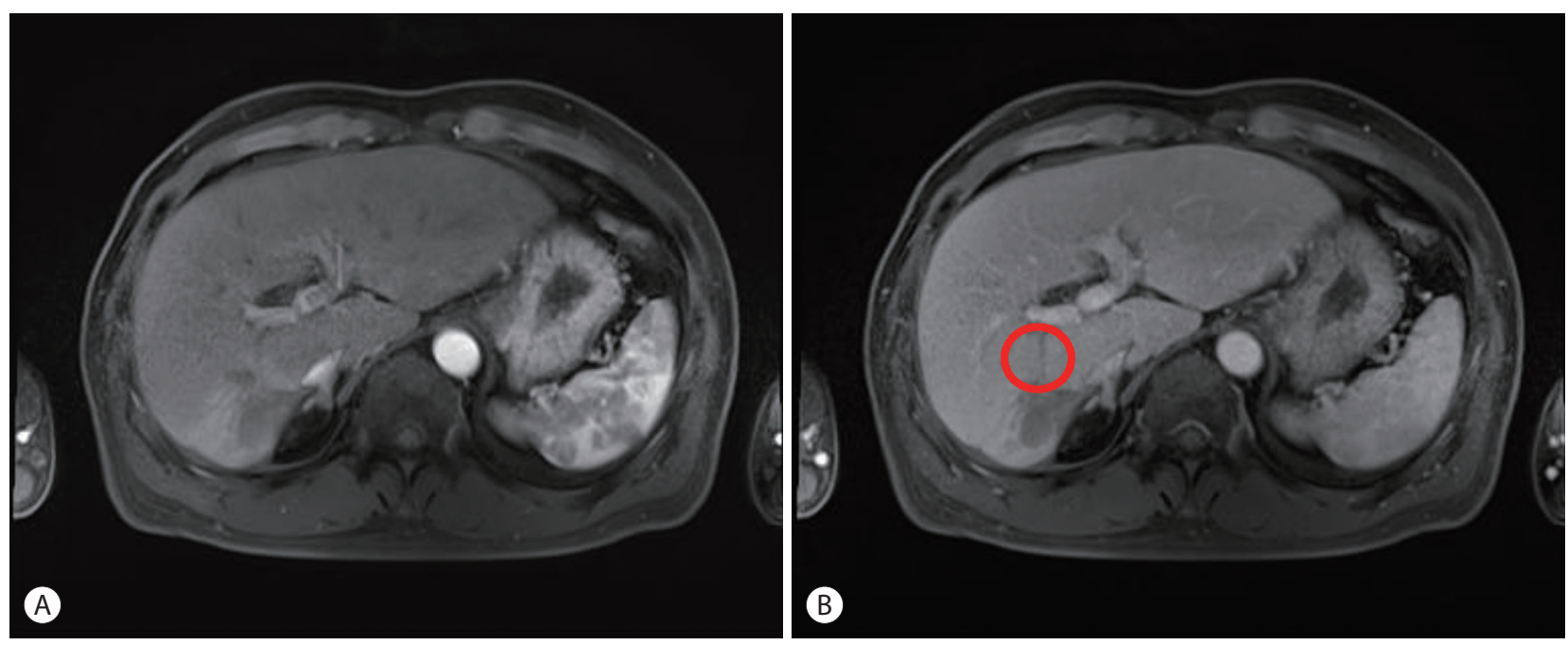

Figure 2. Follow-up liver magnetic resonance imaging (MRI) findings. (A) Arterial phase, (B) portal venous phase. Follow-up liver MRI demonstrated that the largest diameter of the mass was significantly reduced; arterial enhancement was observed only in the periphery of the tumor and internal necrosis was noted (A). The degree of the portal vein tumor thrombus, which initially extended to the right main portal vein, was also reduced to within the right posterior section of the portal vein (circle) (B). 
apy have been used in cases of HCC with PVTT, but the effect is still unsatisfactory.

The prognosis of advanced HCC with PVTT is extremely poor. Few options for treatment are available, and the therapeutic goal is usually limited to partial survival improvement with the prevention of hepatic dysfunction. Sorafenib has been recognized as the standard treatment for advanced HCC since the introduction of the BCLC staging system. The Sorafenib HCC Assessment Randomized Protocol trial demonstrated that sorafenib only modestly increased survival in patients with BCLC stage $\mathrm{C}$ disease from 7.9 months to 10.7 months. ${ }^{14,15}$ Further, in the patients from the Asia Pacific region, the extent of the survival benefits was even lesser than that observed in previous global clinical trials. ${ }^{6,14}$ Jeong et al. ${ }^{16}$ administered sorafenib monotherapy for patients with HCC with PVTT, and reported a median overall survival (OS) of 3.1 months (95\% confidence interval [CI], 2.70-3.50) and a median progression-free survival of 2.0 months (95\% CI, 1.96-2.05). Park ${ }^{17}$ reported that of the 523 patients with advanced HCC who received sorafenib, only seven patients (1.34\%) achieved complete response (CR). Moreover, three of the patients (42.9\%) who achieved CRs eventually experienced recurrence of the disease during the follow-up period. Owing to these limitations of sorafenib, the requirement for alternative therapies has been increasing.

Of the various treatments for advanced HCC with PVTT, HAIC can be an alternative treatment option. HAIC has been applied to treat patients with advanced HCC that was unresectable, the infiltrative type, or in those with PVTT. Theoretically, HAIC shows better efficacy than systemic cytotoxic chemotherapy, because the infusion of the chemotherapeutic agents through the hepatic artery enables direct delivery of high concentrations of drugs to the feeding arteries of the HCC. After selection of the tumor feeding artery, the catheter is inserted at the proper hepatic or common hepatic artery and connected to the port system. The port device in a subcutaneous pocket is implanted in the right or left iliac fossa. HAIC also minimizes systemic toxicities through a greater first-pass effect in the liver, reflecting lower systemic levels of the drugs compared to that in systemic infusion. HAIC has been applied for advanced-stage HCC to improve the therapeutic indices in Asia, especially in Japan and Korea.

Song et al. ${ }^{10}$ determined that the disease control rate of the HAIC group was higher than that of the sorafenib group $(P<0.001)$, and that the OS (7.1 vs. 5.5 months, $P=0.011)$ and time to progression (TTP) $(P=0.034)$ were also significantly longer in the HAIC group. Further, according to the results of a recent randomized clinical trial, the HAIC group achieved better OS and TTP than the sorafenib group did $(P=0.012$ and $P=0.010$, respectively). In multivariate $\operatorname{cox}$ proportional hazard regression models, HAIC appeared to result in a better survival rate than that observed with sorafenib $(P=0.005) .^{8}$ In addition, HAIC resulted in improved survival compared to that observed with sorafenib in patients with Vp3 or Vp4 PVTT. ${ }^{18}$ Moreover, in patients with Vp3 or Vp4 PVTT, HAIC with concurrent irradiation for PVTT was more effective than HAIC alone.

The optimal regimen for HAIC in the treatment of HCC has not yet been established, and a combination of cisplatin and 5-FU was used in our case. Cisplatin is one of the most widely used effective anticancer agents for the treatment of solid tumors. Its mode of action has been linked to its ability to crosslink with the purine bases on DNA, thereby interfering with DNA repair mechanisms, causing DNA damage, and subsequently inducing apoptosis in cancer cells. However, owing to drug resistance and numerous undesirable side effects such as severe nephrotoxicity, allergic reactions, decreased immunity to infections, gastrointestinal hemorrhage, and hearing loss, especially in younger patients, other platinum-containing anticancer drugs have also been used. Further, combination therapies of cisplatin with other drugs have been highly considered to overcome drug resistance and reduce toxicity. ${ }^{19}$ Generally, the treatment cycles are repeated every 4 weeks until disease progression, unacceptable toxicity, or patient refusal to continue.

HAIC is known to have a favorable toxicity profile in patients with advanced HCC. The overall incidence of serious adverse events was significantly higher in the sorafenib group (20.7\%) than in the HAIC group $(6.9 \%) .{ }^{8}$ In addition, discontinuation owing to adverse events was significantly more frequent in the sorafenib group than in the HAIC group. ${ }^{18}$ HAIC was more advantageous than sorafenib in terms of 
side effects.

HCC with vascular invasion typically has a poor prognosis; however, in some cases, downstaging may be successful enough to allow for curative treatment. Further research is required to determine the treatment modalities that can help achieve better outcomes. HAIC can be used as one such downstaging approach to achieve better prognosis in some patients.

\section{ETHICAL APPROVAL}

This study was approved by the Institutional Review Board (KC19ZESE0035).

\section{Conflicts of Interest}

The authors declare that they have no conflicts of interest.

\section{REFERENCES}

1. Heimbach JK, Kulik LM, Finn RS, Sirlin CB, Abecassis MM, Roberts $L R$, et al. AASLD guidelines for the treatment of hepatocellular carcinoma. Hepatology 2018;67:358-380.

2. Pinter M, Peck-Radosavljevic M. Review article: systemic treatment of hepatocellular carcinoma. Aliment Pharmacol Ther 2018;48:598-609.

3. Finn RS, Zhu AX, Farah W, Almasri J, Zaiem F, Prokop LJ, et al. Therapies for advanced stage hepatocellular carcinoma with macrovascular invasion or metastatic disease: a systematic review and meta-analysis. Hepatology 2018;67:422-435.

4. European Association for the Study of the Liver, Galle PR, Forner A, Llovet JM, Mazzaferro V, Piscaglia F, et al. EASL clinical practice guidelines: management of hepatocellular carcinoma. J Hepatol 2018;69:182-236.

5. Omata M, Cheng AL, Kokudo N, Kudo M, Lee JM, Jia J, et al. AsiaPacific clinical practice guidelines on the management of hepatocellular carcinoma: a 2017 update. Hepatol Int 2017;11:317-370.

6. Kim DY, Kim HJ, Han KH, Han SY, Heo J, Woo HY, et al. Real-life experience of sorafenib treatment for hepatocellular carcinoma in Korea: from GIDEON data. Cancer Res Treat 2016;48:1243-1252.

7. Cheng AL, Kang YK, Chen Z, Tsao CJ, Qin S, Kim JS, et al. Efficacy and safety of sorafenib in patients in the Asia-Pacific region with advanced hepatocellular carcinoma: a phase III randomised, double-blind, placebo-controlled trial. Lancet Oncol 2009;10:25-34.

8. Choi JH, Chung WJ, Bae SH, Song DS, Song MJ, Kim YS, et al. Randomized, prospective, comparative study on the effects and safety of sorafenib vs. hepatic arterial infusion chemotherapy in patients with advanced hepatocellular carcinoma with portal vein tumor thrombosis. Cancer Chemother Pharmacol 2018:82:469-478.

9. Onishi H, Nouso K, Nakamura S, Katsui K, Wada N, Morimoto Y, et al. Efficacy of hepatic arterial infusion chemotherapy in combination with irradiation for advanced hepatocellular carcinoma with portal vein invasion. Hepatol Int 2015;9:105-112.

10. Song DS, Bae SH, Song MJ, Lee SW, Kim HY, Lee YJ, et al. Hepatic arterial infusion chemotherapy in hepatocellular carcinoma with portal vein tumor thrombosis. World J Gastroenterol 2013;19:4679-4688.

11. Song MJ. Hepatic artery infusion chemotherapy for advanced hepatocellular carcinoma. World J Gastroenterol 2015;21:3843-3849.

12. Bruix J, Sherman M, American Association for the Study of Liver Diseases. Management of hepatocellular carcinoma: an update. Hepatology 2011;53:1020-1022.

13. Parikh ND, Waljee AK, Singal AG. Downstaging hepatocellular carcinoma: a systematic review and pooled analysis. Liver Transpl 2015;21:1142-1152.

14. Sung PS, Park HL, Yang K, Hwang S, Song MJ, Jang JW, et al. 18Ffluorodeoxyglucose uptake of hepatocellular carcinoma as a prognostic predictor in patients with sorafenib treatment. Eur J Nucl Med Mol Imaging 2018;45:384-391.

15. Llovet JM, Zucman-Rossi J, Pikarsky E, Sangro B, Schwartz M, Sherman $M$, et al. Hepatocellular carcinoma. Nat Rev Dis Primers 2016;2:16018.

16. Jeong SW, Jang JY, Shim KY, Lee SH, Kim SG, Cha SW, et al. Practical effect of sorafenib monotherapy on advanced hepatocellular carcinoma and portal vein tumor thrombosis. Gut Liver 2013;7:696-703.

17. Park JG. Long-term outcomes of patients with advanced hepatocellular carcinoma who achieved complete remission after sorafenib therapy. Clin Mol Hepatol 2015;21:287-294.

18. Moriguchi M, Aramaki T, Nishiofuku H, Sato R, Asakura K, Yamaguchi $K$, et al. Sorafenib versus hepatic arterial infusion chemotherapy as initial treatment for hepatocellular carcinoma with advanced portal vein tumor thrombosis. Liver Cancer 2017;6:275286.

19. Dasari S, Tchounwou PB. Cisplatin in cancer therapy: molecular mechanisms of action. Eur J Pharmacol 2014;740:364-378. 